\title{
ON THE STABILITY OF A JENSEN TYPE FUNCTIONAL EQUATION IN MULTI-NORMED SPACES
}

\author{
KYOO-HONG PARK ${ }^{\mathrm{a}}$ AND YONG-SOO JUnG ${ }^{\mathrm{b}, *}$
}

\begin{abstract}
In this paper we investigate the Hyers-Ulam stability of a Jensen type functional equation in multi-normed spaces and then extend the result to multinormed left modules over a normed algebra $\mathcal{A}$.
\end{abstract}

\section{INTRODUCTION}

The study of stability problems originated from a question by S.M. Ulam [21] in 1940: Under what condition does there exist a homomorphism near an approximate homomorphism? In 1941, D.H. Hyers [8] gave a first affirmative answer to the question of Ulam for Banach spaces, which states that if $\delta>0$ and $f: \mathcal{X} \rightarrow \mathcal{Y}$ is a mapping with $\mathcal{X}$ a normed space, $\mathcal{Y}$ a Banach space such that

$$
\|f(x+y)-f(x)-f(y)\| \leq \delta
$$

for all $x, y \in \mathcal{X}$, then there exists a unique additive mapping $T: \mathcal{X} \rightarrow \mathcal{Y}$ such that

$$
\|f(x)-T(x)\| \leq \delta
$$

for all $x \in \mathcal{X}$.

A generalized version of the theorem of Hyers for approximately additive mappings was first given by T. Aoki [1] in 1950. In 1978, Th.M. Rassias [18] independently introduced the unbounded Cauchy difference and was the first to prove the stability of the linear mapping between Banach spaces

During the past decades, a number of results concerning the stability have been obtained by various ways, and been applied to a number of functional equations and mappings $[3,7,10,19]$.

Received by the editors February 25, 2011. Revised April 26, 2011. Accepted April 29, 2011. 2000 Mathematics Subject Classification. 39B52, 39B72, 39B82.

Key words and phrases. Hyers-Ulam stability, multi-normed space, multi-normed module, Popoviciu's functional equation.

${ }^{*}$ Corresponding author. 
The stability of the classical Jensen functional equation

$$
2 f\left(\frac{x+y}{2}\right)=f(x)+f(y)
$$

and of its generalizations were studied by numerous researchers (cf., e.g., [9, 11, 12, $15])$.

T. Trif [20] studied the generalized Hyers-Ulam stability of the Jensen type functional equation for normed spaces (or is called the Popoviciu functional equation from $[17])$ :

$$
\begin{aligned}
3 f & \left(\frac{x+y+z}{3}\right)+f(x)+f(y)+f(z) \\
& =2\left[f\left(\frac{x+y}{2}\right)+f\left(\frac{y+z}{2}\right)+f\left(\frac{z+x}{2}\right)\right] .
\end{aligned}
$$

In view of [20], we note that the Popoviciu functional equation (1.2) is equivalent to the Jensen functional equation (1.1).

In this paper, using some ideas from the earlier works $[14,16]$, we investigate the stability of the Popoviciu functional equation in multi-normed spaces and further, in multi-normed left module over normed algebra.

The notion of multi-normed space was introduced by H.G. Dales and M.E. Polyakov (see $[5,6,13,14]$ ). This concept is somewhat similar to operator sequence space and has some connections with operator spaces and Banach lattices. Motivations for the study of multi-normed spaces and many examples were given in [6].

Let $\mathbb{C}, \mathbb{R}$ and $\mathbb{N}$ be the sets of complex, real numbers and positive integers, respectively. Let $\mathcal{X}$ be a linear space over $\mathbb{C}$. For each $k \in \mathbb{N}$, we denote by $\mathcal{X}^{k}$ the linear space $\mathcal{X} \oplus \cdots \oplus \mathcal{X}$ consisting of $k$-tuples $\left(x_{1}, \cdots, x_{k}\right)$, where $x_{1}, \cdots, x_{k} \in \mathcal{X}$. The linear operations on $\mathcal{X}^{k}$ are defined coordinatewise. The zero element of either $\mathcal{X}$ or $\mathcal{X}^{k}$ is denoted by 0 . We denote by $\mathbb{N}_{k}$ the set $\{1,2,3, \cdots, k\}$ and by $G_{k}$ the group of permutations on $k$ symbols.

Definition 1.1. A multi-norm on $\left\{\mathcal{X}^{k}: k \in \mathbb{N}\right\}$ is a sequence

$$
\left(\|\cdot\|_{k}\right)=\left(\|\cdot\|_{k}: k \in \mathbb{N}\right)
$$

such that $\|\cdot\|_{k}$ is a norm on $\mathcal{X}^{k}$ for each $k \in \mathbb{N}$, such that $\|x\|_{1}=\|x\|$ for each $x \in \mathcal{X}$, and such that for each $k \in \mathbb{N}(k \geq 2)$, the following axioms are satisfied:

(i) $\left\|x_{\sigma(1)}, \cdots, x_{\sigma(k)}\right\|_{k}=\left\|\left(x_{1}, \cdots, x_{k}\right)\right\|_{k} \quad\left(\sigma \in G_{k} ; x_{1}, \cdots, x_{k} \in \mathcal{X}\right)$;

(ii) $\left\|\left(\alpha_{1} x_{1}, \cdots, \alpha_{k} x_{k}\right)\right\|_{k} \leq\left(\max _{i \in \mathbb{N}_{k}}\left|\alpha_{i}\right|\right)\left\|\left(x_{1}, \cdots, x_{k}\right)\right\|_{k}$ 


$$
\left(\alpha_{1}, \cdots, \alpha_{k} \in \mathbb{C} ; x_{1}, \cdots, x_{k} \in \mathcal{X}\right)
$$

(iii) $\left\|\left(x_{1}, \cdots, x_{k-1}, 0\right)\right\|_{k}=\left\|\left(x_{1}, \cdots, x_{k-1}\right)\right\|_{k-1} \quad\left(x_{1}, \cdots, x_{k-1} \in \mathcal{X}\right)$;

(iv) $\left\|\left(x_{1}, \cdots, x_{k-1}, x_{k-1}\right)\right\|_{k}=\left\|\left(x_{1}, \cdots, x_{k-1}\right)\right\|_{k-1} \quad\left(x_{1}, \cdots, x_{k-1} \in \mathcal{X}\right)$.

In this case, we say that $\left(\left(\mathcal{X}^{k},\|\cdot\|_{k}\right): k \in \mathbb{N}\right)$ is a multi-normed space.

Suppose that $\left(\left(\mathcal{X}^{k},\|\cdot\|_{k}\right): k \in \mathbb{N}\right)$ is a multi-normed space and $k \in \mathbb{N}$. It is easy to show that

(a) $\|(x, \cdots, x)\|_{k}=\|x\| \quad(x \in \mathcal{X})$;

(b) $\max _{i \in \mathbb{N}_{k}}\left\|x_{i}\right\| \leq\left\|\left(x_{1}, \cdots, x_{k}\right)\right\|_{k} \leq \sum_{i=1}^{k}\left\|x_{i}\right\| \leq k \max _{i \in \mathbb{N}_{k}}\left\|x_{i}\right\| \quad\left(x_{1}, \cdots, x_{k} \in \mathcal{X}\right)$.

It follows from (b) that if $(\mathcal{X},\|\cdot\|)$ is a Banach space, then $\left(\mathcal{X}^{k},\|\cdot\|_{k}\right)$ is a Banach space for each $k \in \mathbb{N}$; in this case, $\left(\left(\mathcal{X}^{k},\|\cdot\|_{k}\right): k \in \mathbb{N}\right)$ is said to be a multi-Banach space.

Now we recall two important examples of multi-norms for an arbitrary normed space $\mathcal{X}$ (see, for details, [6]).

Example 1.2. The sequence $\left(\|\cdot\|_{k}: k \in \mathbb{N}\right)$ on $\left\{\mathcal{X}^{k}: k \in \mathbb{N}\right\}$ defined by

$$
\left\|\left(x_{1}, \cdots, x_{k}\right)\right\|_{k}:=\max _{i \in \mathbb{N}_{k}}\left\|x_{i}\right\| \quad\left(x_{1}, \cdots, x_{k} \in \mathcal{X}\right)
$$

is a multi-norm called the minimum multi-norm. The terminology minimum is justified by (b).

Example 1.3. Let $\Lambda$ be a non-empty set and let

$$
\left\{\left(\|\cdot\|_{k}^{\lambda}: k \in \mathbb{N} \text { and } \lambda \in \Lambda\right)\right\}
$$

be the family of all multi-norms on $\left\{\mathcal{X}^{k}: k \in \mathbb{N}\right\}$. For $k \in \mathbb{N}$, we set

$$
\left\|\left(x_{1}, \cdots, x_{k}\right)\right\|_{k}:=\sup _{\lambda \in \Lambda}\left\|\left(x_{1}, \cdots, x_{k}\right)\right\|_{k}^{\lambda} \quad\left(x_{1}, \cdots, x_{k} \in \mathcal{X}\right) .
$$

Then the sequence $\left(\left\||\cdot \||_{k}: k \in \mathbb{N}\right)\right.$ is a multi-norm on $\left\{\mathcal{X}^{k}: k \in \mathbb{N}\right\}$, which is called the maximum multi-norm.

In the following, we need some fundamental ingredients which can be easily deduced from the triangle inequality for the norm $\|\cdot\|_{k}$ and (b).

Definition 1.4. Let $\left(\left(\mathcal{X}^{k},\|\cdot\|_{k}\right): k \in \mathbb{N}\right)$ be a multi-normed space. A sequence $\left(x_{n}\right)$ in $\mathcal{X}$ is a multi-null sequence if, for each $\varepsilon>0$, there exists $n_{0} \in \mathbb{N}$ such that

$$
\sup _{k \in \mathbb{N}}\left\|\left(x_{n}, \cdots, x_{n+k-1}\right)\right\|_{k}<\varepsilon \quad\left(n \geq n_{0}\right) .
$$

Let $x \in \mathcal{X}$. We write that 


$$
\operatorname{Lim}_{n \rightarrow \infty} x_{n}=x
$$

if $\left(x_{n}-x\right)$ is a multi-null sequence; in this case, we say that the sequence $\left(x_{n}\right)$ is multi-convergent to $x$ in $\mathcal{X}$.

Definition 1.5. Let $\left(\left(\mathcal{X}^{k},\|\cdot\|_{k}\right): k \in \mathbb{N}\right)$ be a multi-normed space. A sequence $\left\{x_{n}\right\}$ in $\mathcal{X}$ is a multi-Cauchy sequence if, for each $\varepsilon>0$, there exists $n_{0} \in \mathbb{N}$ such that

$$
\sup _{k \in \mathbb{N}}\left\|\left(x_{m}-x_{n}, \cdots, x_{m+k-1}-x_{n+k-1}\right)\right\|_{k}<\varepsilon \quad\left(m, n \geq n_{0}\right) .
$$

We observe that if $\left(\left(\mathcal{X}^{k},\|\cdot\|_{k}\right): k \in \mathbb{N}\right)$ is a multi-Banach space, then a multiCauchy sequence is multi-convergent in $\mathcal{X}$.

\section{Hyers-Ulam Stability of Equation (1.2) IN Multi-NORMed SPACES}

In this section, $\mathcal{X}$ and $\mathcal{Y}$ will be a complex linear space and a complex Banach space, respectively. Given a function $f: \mathcal{X} \rightarrow \mathcal{Y}$ and $\alpha \in U=\{z \in \mathbb{C}:|z|=1\}$, we set

$$
\begin{aligned}
D_{\alpha} f(x, y, z):= & f\left(\frac{\alpha x+\alpha y+\alpha z}{3}\right)+\alpha f(x)+\alpha f(y)+\alpha f(z) \\
& -2\left[\alpha f\left(\frac{x+y}{2}\right)+f\left(\frac{\alpha y+\alpha z}{2}\right)+f\left(\frac{\alpha z+\alpha x}{2}\right)\right] .
\end{aligned}
$$

Theorem 2.1. Let $\left(\left(\mathcal{Y}^{k},\|\cdot\|_{k}\right): k \in \mathbb{N}\right)$ be a multi-Banach space. If $\delta \geq 0$ and the function $f: \mathcal{X} \rightarrow \mathcal{Y}$ satisfies

$$
\sup _{k \in \mathbb{N}}\left\|\left(D_{1} f\left(x_{1}, y_{1}, z_{1}\right), \cdots, D_{1} f\left(x_{k}, y_{k}, z_{k}\right)\right)\right\|_{k} \leq \delta
$$

for all $x_{1}, \cdots, x_{k}, y_{1}, \cdots, y_{k}, z_{1}, \cdots, z_{k} \in \mathcal{X}$, then there exists a unique additive mapping $A: \mathcal{X} \rightarrow \mathcal{Y}$ such that

$$
\sup _{k \in \mathbb{N}}\left\|\left(f\left(x_{1}\right)-f(0)-A\left(x_{1}\right), \cdots, f\left(x_{k}\right)-f(0)-A\left(x_{k}\right)\right)\right\|_{k} \leq \frac{\delta}{3}
$$

for all $x_{1}, \cdots, x_{k} \in \mathcal{X}$.

Proof. Let $g: \mathcal{X} \rightarrow \mathcal{Y}$ be the function defined by $g(x):=f(x)-f(0)$. Then $g(0)=0$ and, since $D_{1} g(x, y, z)=D_{1} f(x, y, z)$ for all $x, y, z \in \mathcal{X}$, we have

$$
\sup _{k \in \mathbb{N}}\left\|\left(D_{1} g\left(x_{1}, y_{1}, z_{1}\right), \cdots, D_{1} g\left(x_{k}, y_{k}, z_{k}\right)\right)\right\|_{k} \leq \delta
$$

for all $x_{1}, \cdots, x_{k}, y_{1}, \cdots, y_{k}, z_{1}, \cdots, z_{k} \in \mathcal{X}$. 
For each $i=1,2, \cdots, k$, putting $y_{i}=x_{i}$ and $z_{i}=-2 x_{i}$ in (2.3), we get

$$
\sup _{k \in \mathbb{N}}\left\|\left(g\left(-2 x_{1}\right)-4 g\left(-\frac{x_{1}}{2}\right), \cdots, g\left(-2 x_{k}\right)-4 g\left(-\frac{x_{k}}{2}\right)\right)\right\|_{k} \leq \delta
$$

for all $x_{1}, \cdots, x_{k} \in \mathcal{X}$. Replacing $x_{i}$ by $-2 x_{i}$ for each $i=1,2, \cdots, k$ in the above relation yields

$$
\sup _{k \in \mathbb{N}}\left\|\left(g\left(4 x_{1}\right)-4 g\left(x_{1}\right), \cdots, g\left(4 x_{k}\right)-4 g\left(x_{k}\right)\right)\right\|_{k} \leq \delta
$$

for all $x_{1}, \cdots, x_{k} \in \mathcal{X}$.

Next we prove by induction on $n$ that for all $x_{1}, \cdots, x_{k} \in \mathcal{X}$ it holds that

$$
\sup _{k \in \mathbb{N}}\left\|\left(2^{-2 n} g\left(2^{2 n} x_{1}\right)-g\left(x_{1}\right), \cdots, 2^{-2 n} g\left(2^{2 n} x_{k}\right)-g\left(x_{k}\right)\right)\right\|_{k} \leq \delta \sum_{j=1}^{n} 2^{-2 j}
$$

for all $x_{1}, \cdots, x_{k} \in \mathcal{X}$. Dividing both sides of (2.4) by $2^{2}$ ensures the validity of (2.5) for $n=1$. Now, assume that the inequality (2.5) is true for some $n \in \mathbb{N}$. Replacing $x_{i}$ in (2.4) by $2^{2 n} x_{i}$ for each $i=1,2, \cdots, k$ and then dividing both sides of (2.4) by $2^{2(n+1)}$ yields

$$
\begin{aligned}
\sup _{k \in \mathbb{N}} \| & \left(2^{-2(n+1)} g\left(2^{2(n+1)} x_{1}\right)-2^{-2 n} g\left(2^{2 n} x_{1}\right),\right. \\
& \left.\cdots, 2^{-2(n+1)} g\left(2^{2(n+1)} x_{k}\right)-2^{-2 n} g\left(2^{2 n} x_{k}\right)\right) \|_{k} \leq \delta 2^{-2(n+1)}
\end{aligned}
$$

for all $x_{1}, \cdots, x_{k} \in \mathcal{X}$ and so

$$
\begin{aligned}
& \sup _{k \in \mathbb{N}}\left\|\left(2^{-2(n+1)} g\left(2^{2(n+1)} x_{1}\right)-g\left(x_{1}\right), \cdots, 2^{-2(n+1)} g\left(2^{2(n+1)} x_{k}\right)-g\left(x_{k}\right)\right)\right\|_{k} \\
& \leq \sup _{k \in \mathbb{N}} \|\left(2^{-2(n+1)} g\left(2^{2(n+1)} x_{1}\right)-2^{-2 n} g\left(2^{2 n} x_{1}\right),\right. \\
& \left.\quad \cdots, 2^{-2(n+1)} g\left(2^{2(n+1)} x_{k}\right)-2^{-2 n} g\left(2^{2 n} x_{k}\right)\right) \|_{k} \\
& \quad+\sup _{k \in \mathbb{N}}\left\|\left(2^{-2 n} g\left(2^{2 n} x_{1}\right)-g\left(x_{1}\right), \cdots, 2^{-2 n} g\left(2^{2 n} x_{k}\right)-g\left(x_{k}\right)\right)\right\|_{k} \\
& \leq \delta 2^{-2(n+1)}+\delta \sum_{j=1}^{n} 2^{-2 j}=\delta \sum_{j=1}^{n+1} 2^{-2 j}
\end{aligned}
$$

for all $x_{1}, \cdots, x_{k} \in \mathcal{X}$. This completes the proof of the inequality (2.5).

Let $x_{1}, x_{2}, \cdots, x_{k}$ be any points in $\mathcal{X}$. By virtue of (2.5), we have

$$
\begin{gathered}
\sup _{k \in \mathbb{N}}\left\|\left(2^{-2 n} g\left(2^{2 n} x_{1}\right)-2^{-2 m} g\left(2^{2 m} x_{1}\right), \cdots, 2^{-2 n} g\left(2^{2 n} x_{k}\right)-2^{-2 m} g\left(2^{2 m} x_{k}\right)\right)\right\|_{k} \\
\leq 2^{-2 m} \sup _{k \in \mathbb{N}} \|\left(2^{-2(n-m)} g\left(2^{2(n-m)} \cdot 2^{2 m} x_{1}\right)-g\left(2^{2 m} x_{1}\right),\right. \\
\left.\cdots, 2^{-2(n-m)} g\left(2^{2(n-m)} \cdot 2^{2 m} x_{k}\right)-g\left(2^{2 m} x_{k}\right)\right) \|_{k}
\end{gathered}
$$




$$
\leq 2^{-2 m} \delta \sum_{j=1}^{n-m} 2^{-2 j} \leq 2^{-2 m} \frac{\delta}{3} \quad(m<n)
$$

that is,

$$
\begin{aligned}
& \sup _{k \in \mathbb{N}}\left\|\left(2^{-2 n} g\left(2^{2 n} x_{1}\right)-2^{-2 m} g\left(2^{2 m} x_{1}\right), \cdots, 2^{-2 n} g\left(2^{2 n} x_{k}\right)-2^{-2 m} g\left(2^{2 m} x_{k}\right)\right)\right\|_{k} \\
& \leq 2^{-2 m} \frac{\delta}{3} \quad(m<n) .
\end{aligned}
$$

Let us fix $x \in \mathcal{X}$. Then it follows from (2.6) that

$$
\begin{aligned}
& \sup _{k \in \mathbb{N}} \|\left(2^{-2 n} g\left(2^{2 n} x\right)-2^{-2 m} g\left(2^{2 m} x\right),\right. \\
& \left.\quad \cdots, 2^{-2(n+k-1)} g\left(2^{2(n+k-1)} x\right)-2^{-2(m+k-1)} g\left(2^{2(m+k-1)} x\right)\right) \|_{k} \\
& \leq \sup _{k \in \mathbb{N}} \|\left(2^{-2 n} g\left(2^{2 n} x\right)-2^{-2 m} g\left(2^{2 m} x\right),\right. \\
& \left.\quad \cdots, \frac{1}{2^{2(k-1)}}\left(2^{-2 n} g\left(2^{2 n} \cdot 2^{2(k-1)} x\right)-2^{-2 m} g\left(2^{2 m} \cdot 2^{2(k-1)} x\right)\right)\right) \|_{k} \\
& \leq \sup _{k \in \mathbb{N}} \|\left(2^{-2 n} g\left(2^{2 n} x\right)-2^{-2 m} g\left(2^{2 m} x\right),\right. \\
& \left.\quad \cdots, 2^{-2 n} g\left(2^{2 n} \cdot 2^{2(k-1)} x\right)-2^{-2 m} g\left(2^{2 m} \cdot 2^{2(k-1)} x\right)\right) \|_{k} \leq 2^{-2 m} \frac{\delta}{3} \quad(m<n) .
\end{aligned}
$$

This inequality implies that $\left\{2^{-2 n} g\left(2^{2 n} x\right)\right\}$ is a multi-Cauchy sequence and so it is multi-convergent in $\mathcal{Y}$. Consequently, we can define the mapping $A: \mathcal{X} \rightarrow \mathcal{Y}$ by

$$
A(x):=\operatorname{Lim}_{n \rightarrow \infty} 2^{-2 n} g\left(2^{2 n} x\right) .
$$

Therefore, for any $\varepsilon>0$, there exists $n_{0} \in \mathbb{N}$ such that

$$
\sup _{k \in \mathbb{N}}\left\|\left(2^{-2 n} g\left(2^{2 n} x\right)-A(x), \cdots, 2^{-2(n+k-1)} g\left(2^{2(n+k-1)} x\right)-A(x)\right)\right\|_{k}<\varepsilon
$$

for all $n \geq n_{0}$. In particular, by (b), we have

$$
\lim _{n \rightarrow \infty}\left\|2^{-2 n} g\left(2^{2 n} x\right)-A(x)\right\|=0,
$$

say,

$$
A(x)=\lim _{n \rightarrow \infty} 2^{-2 n} g\left(2^{2 n} x\right)
$$

for all $x \in \mathcal{X}$.

Let $x, y$ and $z$ be any points in $\mathcal{X}$. Putting $x_{1}=\cdots=x_{k}=2^{2 n} x, y_{1}=\cdots=$ $y_{k}=2^{2 n} y$ and $z_{1}=\cdots=z_{k}=2^{2 n} z$ in $(2.3)$ and dividing both sides by $2^{2 n}$, we obtain 


$$
\begin{aligned}
\left\|D_{1} A(x, y, z)\right\| & =\lim _{n \rightarrow \infty} 2^{-2 n}\left\|D_{1} g\left(2^{2 n} x, 2^{2 n} y, 2^{2 n} z\right)\right\| \\
& \leq \lim _{n \rightarrow \infty} 2^{-2 n} \delta=0 .
\end{aligned}
$$

Hence $A$ satisfies (1.2) for all $x, y, z \in \mathcal{X}$. Since $A(0)=0$, it follows that $A$ is additive. Moreover, by passing to the limit in (2.5) when $n \rightarrow \infty$, we see that

$$
\sup _{k \in \mathbb{N}}\left\|\left(A\left(x_{1}\right)-g\left(x_{1}\right), \cdots, A\left(x_{k}\right)-g\left(x_{k}\right)\right)\right\|_{k} \leq \frac{\delta}{3}
$$

which means the inequality $(2.2)$ for all $x_{1}, \cdots, x_{k} \in \mathcal{X}$.

Let $A^{\prime}: \mathcal{X} \rightarrow \mathcal{Y}$ be another additive mapping satisfying (2.2). Using (2.2) and (a), we get

$$
\begin{aligned}
\left\|A(x)-A^{\prime}(x)\right\| & =2^{-n}\left\|A\left(2^{n} x\right)-A^{\prime}\left(2^{n} x\right)\right\| \\
& \leq 2^{-n}\left(\left\|A\left(2^{n} x\right)-f\left(2^{n} x\right)-f(0)\right\|+\left\|f\left(2^{n} x\right)-f(0)-A^{\prime}\left(2^{n} x\right)\right\|\right) \\
& \leq 2^{-n} \frac{2}{3} \delta .
\end{aligned}
$$

for all $x \in \mathcal{X}$ and $n \in \mathbb{N}$. Thus we conclude that $A(x)=A^{\prime}(x)$ for all $x \in \mathcal{X}$. This proves the uniqueness of $A$.

Theorem 2.2. Let $\left(\left(\mathcal{Y}^{k},\|\cdot\|_{k}\right): k \in \mathbb{N}\right)$ be a multi-Banach space. If $\delta \geq 0$ and the function $f: \mathcal{X} \rightarrow \mathcal{Y}$ satisfies

$$
\sup _{k \in \mathbb{N}}\left\|\left(D_{\alpha} f\left(x_{1}, y_{1}, z_{1}\right), \cdots, D_{\alpha} f\left(x_{k}, y_{k}, z_{k}\right)\right)\right\|_{k} \leq \delta
$$

for all $x_{1}, \cdots, x_{k}, y_{1}, \cdots, y_{k}, z_{1}, \cdots, z_{k} \in \mathcal{X}$ and for all $\alpha \in U$, then there exists a unique $\mathbb{C}$-linear mapping $A: \mathcal{X} \rightarrow \mathcal{Y}$ satisfying the inequality (2.2).

Proof. Put $\alpha=1$ in (2.7). Then it follows from Theorem 2.1 that there exists a unique additive mapping $A: \mathcal{X} \rightarrow \mathcal{Y}$ satisfying the inequality (2.2).

For each $i=1,2, \cdots, k$, setting $y_{i}=x_{i}=x$ and $z_{i}=-2 x$ in (2.7) and then considering (a), we get

$$
\left\|3 f(0)+\alpha f(-2 x)-4 f\left(-\frac{\alpha}{2} x\right)\right\| \leq \delta
$$

for all $x \in \mathcal{X}$. Put $\varepsilon:=\delta+3\|f(0)\|$. From (2.8) and the triangle inequality, it follows that

$$
\left\|\alpha f(-2 x)-4 f\left(-\frac{\alpha}{2} x\right)\right\| \leq \varepsilon
$$

for all $x \in \mathcal{X}$. Substituting $-2 x$ for $x$ in (2.9) yields

$$
\|\alpha f(4 x)-4 f(\alpha x)\| \leq \varepsilon
$$


for all $x \in \mathcal{X}$. Using induction on $n \in \mathbb{N}$ with (2.10), we see that

$$
\left\|\alpha f\left(2^{2 n} x\right)-4 f\left(2^{2(n-1)} \alpha x\right)\right\| \leq \varepsilon
$$

for all $x \in \mathcal{X}$.

Now letting $\alpha=1$ in (2.11) and then replacing $x$ by $\alpha x$ in the result, we obtain

$$
\left\|f\left(2^{2 n} \alpha x\right)-4 f\left(2^{2(n-1)} \alpha x\right)\right\| \leq \varepsilon
$$

for all $x \in \mathcal{X}$. By (2.11) and (2.12), we get

$$
\begin{aligned}
\left\|f\left(2^{2 n} \alpha x\right)-\alpha f\left(2^{2 n} x\right)\right\| \leq & \left\|f\left(2^{2 n} \alpha x\right)-4 f\left(2^{2(n-1)} \alpha x\right)\right\| \\
& +\left\|\alpha f\left(2^{2 n} x\right)-4 f\left(2^{2(n-1)} \alpha x\right)\right\| \leq 2 \varepsilon,
\end{aligned}
$$

that is,

$$
\left\|f\left(2^{2 n} \alpha x\right)-\alpha f\left(2^{2 n} x\right)\right\| \leq 2 \varepsilon .
$$

for all $x \in \mathcal{X}$ which implies

$$
\lim _{n \rightarrow \infty} 2^{-2 n}\left\|f\left(2^{2 n} \alpha x\right)-\alpha f\left(2^{2 n} x\right)\right\|=0,
$$

for all $x \in \mathcal{X}$. Hence we conclude that

$$
A(\alpha x)=\lim _{n \rightarrow \infty} 2^{-2 n} f\left(2^{2 n} \alpha x\right)=\lim _{n \rightarrow \infty} 2^{-2 n} \alpha f\left(2^{2 n} x\right)=\alpha A(x)
$$

for all $\alpha \in U$ and $x \in \mathcal{X}$.

Clearly, $A(0 x)=0=0 A(x)$ for all $x \in \mathcal{X}$. Now, let $\lambda \in \mathbb{C}(\lambda \neq 0)$, and let $N \in \mathbb{N}$ greater than $|\lambda|$. By applying a geometric argument, we see that there exists $\lambda_{1}, \lambda_{2} \in U$ such that $2 \frac{\lambda}{N}=\lambda_{1}+\lambda_{2}$. By the additivity of $A$, we get $A\left(\frac{1}{2} x\right)=\frac{1}{2} A(x)$ for all $x \in \mathcal{X}$. Therefore

$$
\begin{aligned}
A(\lambda x) & =A\left(\frac{N}{2} \cdot 2 \cdot \frac{\lambda}{N} x\right)=N A\left(\frac{1}{2} \cdot 2 \cdot \frac{\lambda}{N} x\right)=\frac{N}{2} A\left(\left(\lambda_{1}+\lambda_{2}\right) x\right) \\
& =\frac{N}{2}\left(\lambda_{1}+\lambda_{2}\right) A(x)=\frac{N}{2} \cdot 2 \cdot \frac{\lambda}{N} A(x)=\lambda A(x)
\end{aligned}
$$

for all $x \in \mathcal{A}$, so that $A$ is $\mathbb{C}$-linear.

\section{Stability of Equation (1.2) in Multi-normed Modules}

In this section, we extend the Hyers-Ulam stability of the Popoviciu's functional equation to multi-normed left modules over a normed algebra and obtain some related results. For the sake of convenience, we use the same symbol $\|\cdot\|$ in order to represent the norms on a normed algebra and a normed module.

Consider first some definitions and examples: 
Definition 3.1. Let $(\mathcal{A},\|\cdot\|)$ be a normed algebra such that $\left(\left(\mathcal{A}^{k},\|\cdot\|_{k}\right): k \in \mathbb{N}\right)$ is a multi-normed space. $\left(\left(\mathcal{A}^{k},\|\cdot\|_{k}\right): k \in \mathbb{N}\right)$ is called a multi-normed algebra if

$$
\left\|\left(a_{1} b_{1}, \cdots, a_{k} b_{k}\right)\right\|_{k} \leq\left\|\left(a_{1}, \cdots, a_{k}\right)\right\|_{k}\left\|\left(b_{1}, \cdots, b_{k}\right)\right\|_{k}
$$

for $k \in \mathbb{N}$ and $a_{1}, \cdots, a_{k}, b_{1}, \cdots, b_{k} \in \mathcal{A}$. Furthermore, the multi-normed algebra $\left(\left(\mathcal{A}^{k},\|\cdot\|_{k}\right): k \in \mathbb{N}\right)$ is said to be a multi-Banach algebra if $\left(\left(\mathcal{A}^{k},\|\cdot\|_{k}\right): k \in \mathbb{N}\right)$ is a multi-Banach space.

Example 3.2. Let $p, q \in \mathbb{R}$ with $1 \leq p \leq q<\infty$ and $\mathcal{A}=\ell^{p}$. The algebra $\mathcal{A}$ is a Banach sequence algebra with respect to coordinatewise multiplication of sequences (see [4, Example 4.2.42]). Let $\left(\|\cdot\|_{k}: k \in \mathbb{N}\right)$ be the standard $(p, q)$-multi-norm on $\left\{A^{k}: n \in \mathbb{N}\right\}$ (see [6]). Then $\left(\left(\mathcal{A}^{k},\|\cdot\|_{k}\right): k \in \mathbb{N}\right)$ is a multi-Banach algebra.

Definition 3.3. Let $(\mathcal{A},\|\cdot\|)$ be a normed algebra. Let $(\mathcal{M},\|\cdot\|)$ be a normed left $\mathcal{A}$ module such that $\left(\left(\mathcal{M}^{k},\|\cdot\|_{k}\right): k \in \mathbb{N}\right)$ is a multi-normed space. $\left(\left(\mathcal{M}^{k},\|\cdot\|_{k}\right): k \in \mathbb{N}\right)$ is said to be a multi-normed left $\mathcal{A}$-module if there exists a positive constant $K$ such that $\left\|\left(a x_{1}, \cdots, a x_{k}\right)\right\|_{k} \leq K\|a\|\left\|\left(x_{1}, \cdots, x_{k}\right)\right\|_{k}$ for all $a \in \mathcal{A}$ and $x_{1}, \cdots, x_{k} \in \mathcal{M}$. Moreover, we says that the multi-normed left $\mathcal{A}$-module $\left(\left(\mathcal{M}^{k},\|\cdot\|_{k}\right): k \in \mathbb{N}\right)$ is a multi-Banach left $\mathcal{A}$-module if $\left(\left(\mathcal{M}^{k},\|\cdot\|_{k}\right): k \in \mathbb{N}\right)$ is a multi-Banach space.

Example 3.4. Let $(\mathcal{A},\|\cdot\|)$ be a normed algebra and $\left(\left(\mathcal{A}^{k},\|\cdot\|_{k}\right): k \in \mathbb{N}\right)$ a multi-normed algebra. Then $\left(\left(\mathcal{A}^{k},\|\cdot\|_{k}\right): k \in \mathbb{N}\right)$ is a multi-normed left $\mathcal{A}$-module.

Example 3.5. Let $(\mathcal{A},\|\cdot\|)$ be a normed algebra and $\left(\left(\mathcal{A}^{k},\|\cdot\|_{k}\right): k \in \mathbb{N}\right)$ a multi-normed algebra. Let $I$ be a closed left ideal of $\mathcal{A}$, let $\mathcal{M}=\mathcal{A} / I$, and let $a \mapsto a+I$ denote the canonical mapping of $\mathcal{A}$ onto $\mathcal{M}$. Then the normed linear space $(\mathcal{M},\|\cdot\|)$ becomes a normed left $\mathcal{A}$-module with the module multiplication given by $a x=a b+I$, where $b \in x \in \mathcal{M}, a \in \mathcal{A}$. Now, it is easy to see that $\left(\left(\mathcal{M}^{k},\|\cdot\|_{k}\right): k \in \mathbb{N}\right)$ is a multi-normed left $\mathcal{A}$-module.

Definition 3.6. Let $\mathcal{A}$ be an algebra. A left $\mathcal{A}$-module $\mathcal{M}$ is said to be unitary if $\mathcal{A}$ has a unit element $e$ and $e x=x$ for all $x \in \mathcal{M}$.

Throughout this section, let $(\mathcal{A},\|\cdot\|)$ be a unital normed algebra with unit $e, \mathcal{M}_{1}$ a unitary left $\mathcal{A}$-module and $\left(\mathcal{M}_{2},\|\cdot\|\right)$ a unitary Banach left $\mathcal{A}$-module.

Recall [16] that an additive mapping $f: \mathcal{M}_{1} \rightarrow \mathcal{M}_{2}$ is said to be $\mathcal{A}$-linear if $f(a x)=a f(x)$ for all $a \in \mathcal{A}$ and $x \in \mathcal{M}_{1}$.

Given a function $f: \mathcal{M}_{1} \rightarrow \mathcal{M}_{2}$ and $a \in \mathcal{A}$ with $\|a\|=1$, we put 


$$
\begin{aligned}
D_{a} f(x, y, z):=3 f( & \left.\frac{a x+a y+a z}{3}\right)+a f(x)+a f(y)+a f(z) \\
& -2\left[a f\left(\frac{x+y}{2}\right)+f\left(\frac{a y+a z}{2}\right)+f\left(\frac{a z+a x}{2}\right)\right]
\end{aligned}
$$

for all $x, y, z \in \mathcal{M}_{1}$.

Theorem 3.7. Let $\left(\left(\mathcal{M}_{2}^{k},\|\cdot\|_{k}\right): k \in \mathbb{N}\right)$ be a multi-Banach left $\mathcal{A}$-module. If $\delta \geq 0$ and the function $f: \mathcal{M}_{1} \rightarrow \mathcal{M}_{2}$ satisfies

$$
\sup _{k \in \mathbb{N}}\left\|\left(D_{a} f\left(x_{1}, y_{1}, z_{1}\right), \cdots, D_{a} f\left(x_{k}, y_{k}, z_{k}\right)\right)\right\|_{k} \leq \delta
$$

for all $x_{1}, \cdots, x_{k}, y_{1}, \cdots, y_{k}, z_{1}, \cdots, z_{k} \in \mathcal{M}_{1}$ and for all $a \in \mathcal{A}$ with $\|a\|=1$, then there exists a unique $\mathcal{A}$-linear mapping $A: \mathcal{M}_{1} \rightarrow \mathcal{M}_{2}$ satisfying the inequality (2.2).

Proof. Using Theorem 2.2, it follows from the inequality (3.1) for $a=\alpha e, \alpha \in U$, that there exists a unique $\mathbb{C}$-linear mapping $A: \mathcal{M}_{1} \rightarrow \mathcal{M}_{2}$ defined by

$$
A(x)=\lim _{n \rightarrow \infty} 2^{-2 n} f\left(2^{2 n} x\right)
$$

for all $x \in \mathcal{M}_{1}$ such that the inequality (2.2) is valid.

The substitution $a$ for $\alpha$ in (2.8) (2.13) and the same process yield

$$
\left\|f\left(2^{2 n} a x\right)-a f\left(2^{2 n} x\right)\right\| \leq 2 \varepsilon
$$

for all $x \in \mathcal{M}_{1}$ which gives

$$
\lim _{n \rightarrow \infty} 2^{-2 n}\left\|f\left(2^{2 n} a x\right)-a f\left(2^{2 n} x\right)\right\|=0,
$$

for all $x \in \mathcal{M}_{1}$. Thus, we see that

$$
A(a x)=\lim _{n \rightarrow \infty} 2^{-2 n} f\left(2^{2 n} a x\right)=\lim _{n \rightarrow \infty} 2^{-2 n} a f\left(2^{2 n} x\right)=a A(x)
$$

for all $a \in \mathcal{A}$ with $\|a\|=1$ and all $x \in \mathcal{M}_{1}$. Since $A$ is $\mathbb{C}$-linear and $A(a x)=a A(x)$ for each element $a \in \mathcal{A}$ with $\|a\|=1$, we have, for all $a \in \mathcal{A} \backslash\{0\}$ and all $x \in \mathcal{M}_{1}$,

$$
A(a x)=A\left(\|a\| \frac{a}{\|a\|} x\right)=\|a\| A\left(\frac{a}{\|a\|} x\right)=\|a\| \frac{a}{\|a\|} A(x)=a A(x) .
$$

Therefore, the unique $\mathbb{C}$-linear mapping $A: \mathcal{M}_{1} \rightarrow \mathcal{M}_{2}$ is an $\mathcal{A}$-linear mapping, as desired.

Theorem 3.8. Let $\mathcal{A}$ be a Banach $*$-algebra, $\operatorname{pos}(\mathcal{A})$ the set of positive elements of $\mathcal{A}$ and $\left(\left(\mathcal{M}_{2}^{k},\|\cdot\|_{k}\right): k \in \mathbb{N}\right)$ a multi-Banach left $\mathcal{A}$-module. If the function $f: \mathcal{M}_{1} \rightarrow$ $\mathcal{M}_{2}$ satisfies the inequality (3.1) for all $x_{1}, \cdots, x_{k}, y_{1}, \cdots, y_{k}, z_{1}, \cdots, z_{k} \in \mathcal{M}_{1}$ and all $a \in \operatorname{pos}(\mathcal{A})$ with $\|a\|=1$ and $a=i$, then there exists a unique $\mathcal{A}$-linear mapping $A: \mathcal{M}_{1} \rightarrow \mathcal{M}_{2}$ satisfying the inequality (2.2). 
Proof. By the same reasoning as in the proof of Theorem 2.2, there exists a unique additive mapping $A: \mathcal{M}_{1} \rightarrow \mathcal{M}_{2}$ defined by $A(x)=\lim _{n \rightarrow \infty} 2^{-2 n} f\left(2^{2 n} x\right)$ for all $x \in \mathcal{M}_{1}$ such that the inequality (2.2) holds. Following the same method as in the proof of Theorem 3.7, we see that

$$
A(a x)=\lim _{n \rightarrow \infty} 2^{-2 n} f\left(2^{2 n} a x\right)=\lim _{n \rightarrow \infty} 2^{-2 n} a f\left(2^{2 n} x\right)=a A(x)
$$

for all $a \in \operatorname{pos}(\mathcal{A})$ with $\|a\|=1$ or $a=i$, and $x \in \mathcal{M}_{1}$. For any element $a \in \mathcal{A}$, $a=a_{1}+i a_{2}$, where $a_{1}=\frac{a+a^{*}}{2}$ and $a_{2}=\frac{a-a^{*}}{2 i}$ are self-adjoint elements, furthermore, $a=a_{1}{ }^{+}-a_{1}{ }^{-}+i a_{2}{ }^{+}-i a_{2}{ }^{-}$, where $a_{1}{ }^{+}, a_{1}{ }^{-}, a_{2}{ }^{+}, a_{2}{ }^{-} \in \operatorname{pos}(\mathcal{A})$ (see [2, Lemma 38.8]). Therefore, we have

$$
\begin{aligned}
A(a x) & =A\left(a_{1}{ }^{+} x-{a_{1}}^{-} x+i a_{2}{ }^{+} x-i a_{2}{ }^{-} x\right) \\
& =a_{1}{ }^{+} A(x)-a_{1}{ }^{-} A(x)+a_{2}{ }^{+} A(i x)-a_{2}{ }^{-} A(i x) \\
& =a_{1}{ }^{+} A(x)-a_{1}{ }^{-} A(x)+i a_{2}{ }^{+} A(x)-i a_{2}{ }^{-} A(x) \\
& =\left(a_{1}{ }^{+}-a_{1}{ }^{-}+i a_{2}{ }^{+}-i a_{2}{ }^{-}\right) A(x) \\
& =a A(x)
\end{aligned}
$$

for all $a \in \mathcal{A}$ and all $x \in \mathcal{M}_{1}$ which completes the proof of the theorem.

\section{REFERENCES}

1. T. Aoki: On the stability of the linear transformation in Banach spaces. J. Math. Soc. Japan 2 (1950), 64-66.

2. F.F. Bonsall \& J. Duncan: Complete normed algebras. Berlin-Heidelberg-New York, 1973.

3. S. Czerwik: On the stability of the quadratic mapping in normed spaces. Abh. Math. Sem. Univ. Hamburg 62 (1992), 59-64.

4. H.G. Dales: Banach Algebras and Automatic Continuity. London Mathematical Society Monographs, New Series, 24. Oxford University Press, Oxford, 2000.

5. H.G. Dales \& M.S. Moslehian: Stability of mappings on multi-normed spaces. Glasgow Math. J. 49 (2007), no. 2, 321-332.

6. H.G. Dales \& M.E. Polyakov: Multi-normed spaces and multi-Banach algebras. preprint.

7. P. Găvruţă: A generalization of the Hyers-Ulam-Rassias Stability of approximately additive mappings. J. Math. Anal. Appl. 184 (1994), 431-436.

8. D.H. Hyers: On the stability of the linear functional equation. Proc. Natl. Acad. Sci. 27 (1941), 222-224.

9. S.-M. Jung: Hyers-Ulam-Rassias stability of Jensens equation and its application. Proc. Amer. Math. Soc. 126 (1998), 3137-3143. 
10. ___ Hyers-Ulam-Rassias Stability of Functional equations in Mathematical Analysis. Hadronic Press, Inc., Palm Harbor, Florida, 2001.

11. Z. Kominek: On a local stability of the Jensen functional equation. Demonstratio Math. 22 (1989), 499-507.

12. Y.-H. Lee \& K.-W. Jun: A generalization of the Hyers-Ulam-Rassias stability of Jensens equation. J. Math. Anal. Appl. 238 (1999), 305-315.

13. M.S. Moslehian, K. Nikodem \& D. Popa: Asymptotic aspect of the quadratic functional equation in multi-normed spaces. J. Math. Anal. and Appl. 355 (2009), no. 2, 717-724.

14. M.S. Moslehian: Superstability of higher derivations in multi-Banach algebras. Tamsui Oxford J. Math. Sciences 24 (2008), no. 4, 417-427.

15. L. Li, J. Chung \& D. Kim: Stability of Jensen equations in the space of generalized functions. J. Math. Anal. Appl. 299 (2004), 578-586.

16. C. Park: On the stability of the linear mapping in Banach modules. J. Math. Anal. Appl. 275 (2002), 711-720.

17. T. Popoviciu: Sur certaines inégalités qui caractérisent les fonctions convexes. Ştiint. Univ. Al. I. Cuza Iaşi Sect. Ia Mat. 11 (1965), 155-164.

18. Th.M. Rassias: On the stability of the linear mapping in Banach spaces. Proc. Amer. Math. Soc. 72 (1978), 297-300.

19. (Ed.): "Functional Equations and Inequalities". Kluwer Academic, Dordrecht, Boston, London, 2000.

20. T. Trif: Hyers-Ulam-Rassias stability of a Jensen type functional equation. J. Math. Anal. Appl. 250 (2000), 579-588

21. S.M. Ulam: A Collection of Mathematical Problems. Interscience Publ., New York, 1960 .

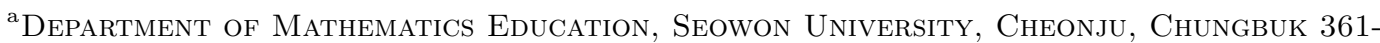
742, KOREA

Email address: parkkh@seowon.ac.kr

${ }^{\text {b} D e p a r t m e n t ~ o f ~ M a t h e m a t i c s, ~ S u n ~ M o o n ~ U n i v e r s i t y, ~ A s a n, ~ C h u n g n a m ~ 336-708, ~ K o r e a ~}$

Email address: ysjung@sunmoon.ac.kr 